\title{
Factorization in high-energy nucleus-nucleus fragmentation cross sections
}

\author{
J. Cugnon* and R. Sartor \\ Institut de Physique, Université de Liège, Sart Tilman, B-4000 Liège 1, Belgique \\ (Received 17 September 1979)
}

It is shown that the basic quantity of the abrasion-ablation model of nucleus-nucleus collisions, namely the probability of extracting a nucleon from the projectile, is a quasi-universal quantity. It can be given a form which does not depend upon the target and which depends weakly upon the projectile. It is shown how this property is related to the weak factorization of the cross sections.

[NUCLEAR REACTIONS Fragmentation in relativistic heavy ion collisions.] Factorization of the cross section.

It has been observed for a few years $\mathrm{s}^{1,2}$ that the inclusive fragmentation cross sections in the relativistic nucleus-nucleus collisions can be factorized according to the formula

$$
\sigma(\mathrm{F}, \mathrm{P}, \mathrm{T})=\sigma_{\mathrm{P}}^{\mathrm{F}} \gamma_{\mathrm{PT}},
$$

where $F, P$, and $T$ stand for the fragment, the projectile, and the target, respectively. Form (1) can be called weak factorization as opposed to the strong factorization form which reads

$$
\sigma(\mathrm{F}, \mathrm{P}, \mathrm{T})=\sigma_{\mathrm{P}}^{\mathrm{F}} \gamma_{\mathrm{T}} \text {. }
$$

The present experimental data do not allow one to draw conclusions about the validity of relation (2).

It has been shown recently ${ }^{3}$ that the abrasionablation model $^{4-8}$ of relativistic heavy ion collisions predicts that weak factorization is approximately valid while strong factorization is violated. In this paper, our aim is to bring to light the exact relation between the abrasion-ablation model and the factorization and to comment about some properties of the model.

Let us call $\sigma_{n}(\mathrm{P}, \mathrm{T})$ the abrasion cross section for removing $n$ nucleons from the projectile. It is clear that the factorization of $\sigma_{n}(\mathrm{P}, \mathrm{T})$ implies the one of the abrasion-ablation cross section (see Ref. 5). One has the basic relations ${ }^{5}$

$$
\begin{aligned}
\sigma_{n}(\mathrm{P}, \mathrm{T})=\left(\begin{array}{c}
A_{\mathrm{P}} \\
n
\end{array}\right) \int_{0}^{\infty} & d^{2} \overrightarrow{\mathrm{b}}\left[1-P_{\mathrm{PT}}(b)\right]^{n} \\
& \times\left[P_{\mathrm{PT}}(b)\right]^{A_{\mathrm{P}}-n},
\end{aligned}
$$

where the probability function $P_{\mathrm{PT}}(b)$ is defined by

$$
P_{\mathrm{PT}}(b)=\int d^{2} \overrightarrow{\mathrm{t}} \phi_{\mathrm{P}}(\overrightarrow{\mathrm{t}}) \exp \left[-A_{\mathrm{T}} \sigma_{\mathrm{NN}} \phi_{\mathrm{T}}(\overrightarrow{\mathrm{t}}+\overrightarrow{\mathrm{b}})\right]
$$

with

$$
\phi_{i}(\overrightarrow{\mathrm{t}})=\int_{-\infty}^{+\infty} d z \rho_{i}(\overrightarrow{\mathrm{t}}, z), \quad i=\mathrm{P}, \mathrm{T} .
$$

In the above equations, $\sigma_{\mathrm{NN}}$ is the nucleon-nu- cleon cross section and $\rho$ is the nuclear density. $(\overrightarrow{\mathrm{t}}, z)$ are cylindrical coordinates with $z$ along the incident direction and $\vec{b}$ is the impact parameter.

We recall that Eqs. (3)-(5) are obtained by using the nonrelativistic Glauber multiple scattering theory ${ }^{9}$ as applied ${ }^{10-17}$ to a composite projectile and target. It is interesting to note that the trivial relativistic extension, namely the introduction of Lor entz contracted objects, does not change the value of $P_{P_{T}}(b)$ since the function $\phi(\vec{t})$ is not affected by a Lorentz contraction.

We have computed the quantities $\sigma_{n}(\mathrm{P}, \mathrm{T})$ and $P_{\mathrm{PT}}(b)$ for a large number of colliding pairs. Our results confirm those of Ref. 3. Moreover, we observed that the probability function $P_{\mathrm{PT}}(b)$ can be written

$$
P_{\mathrm{PT}}(b)=f_{\mathrm{P}}\left(b-\xi_{\mathrm{T}}\right),
$$

where the function $f_{\mathrm{P}}(x)$ only depends upon the projectile $\mathrm{P}$. In other words, the quantity $P_{\mathrm{PT}}(b)$ only depends on the target $\mathrm{T}$ by a translation of the coordinate. More remarkable perhaps is the fact that the amplitude $\xi_{\mathrm{T}}$ of the translation does not depend upon the projectile (see Table I). We show in Fig. 1 how the relation (6) is fulfilled for the

\begin{tabular}{|c|c|c|c|c|c|c|}
\hline & $\xi$ & ${ }^{16} \mathrm{O}$ & ${ }^{27} \mathrm{Al}$ & $\begin{array}{c}\mathrm{T} \\
{ }^{63} \mathrm{Cu}\end{array}$ & ${ }^{107} \mathrm{Ag}$ & ${ }^{208} \mathrm{~Pb}$ \\
\hline \multirow{5}{*}{$\mathrm{P}$} & ${ }^{16} \mathrm{O}$ & -2.1 & -1.3 & 0 & 1.1 & 2.6 \\
\hline & ${ }^{27} \mathrm{Al}$ & -2 & -1.3 & 0 & 1 & 2.5 \\
\hline & ${ }^{63} \mathrm{Cu}$ & -2.1 & -1.3 & 0 & 1 & 2.5 \\
\hline & ${ }^{107} \mathrm{Ag}$ & -2.1 & -1.3 & 0 & 1.1 & 2.6 \\
\hline & ${ }^{208} \mathrm{~Pb}$ & -2.1 & -1.3 & 0 & 1.1 & 2.6 \\
\hline
\end{tabular}
${ }^{27} \mathrm{Al}$ and ${ }^{107} \mathrm{Ag}$ projectiles. The discrepancy for $x=b-\xi_{\mathrm{T}}$ smaller than $5 \mathrm{fm}$ which arises for light targets only can be disregarded in practice since

TABLE I. The translation amplitude $\xi$ (in $\mathrm{fm}$ ) of Eq. (6) for different projectiles (P) and targets (T). It appears that $\xi$ is a function of $\mathrm{T}$ only. 


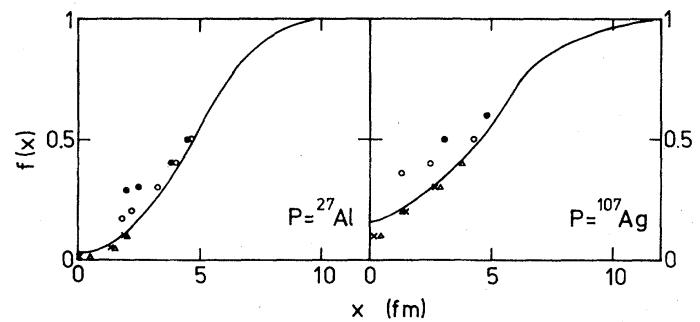

FIG. 1. The universal function $f_{P}(x)$ for the ${ }^{27} \mathrm{Al}$ and ${ }^{107} \mathrm{Ag}$ projectiles. $f_{P}(x)$ is the probability function $P_{\mathrm{PT}}(x)$ for the ${ }^{63} \mathrm{Cu}$ target. The filled $(\bullet)$ and open $(O)$ dots, the crosses $(X)$ and the triangles $(\triangle)$ correspond to the translated probability functions of the ${ }^{16} \mathrm{O},{ }^{27} \mathrm{Al},{ }^{107} \mathrm{Ag}$, and ${ }^{208} \mathrm{~Pb}$ targets, respectively. For $x$ greater than 5 $\mathrm{fm}$, they all fall on the universal curve and are not shown. The computations were done at an energy of 2 $\mathrm{GeV} /$ nucleon with a Woods-Saxon density distribution for the targets and projectiles.

the main contribution to the integral in Eq. (3) comes from higher values of $x$ [see Eq. (7) below]. We have set $\xi_{\mathrm{T}}$ equal to zero for the ${ }^{63} \mathrm{Cu}$ target.

Let us now show how the above mentioned quasiuniversality guarantees the weak factorization property of the cross sections. By making use of (6) Eq. (3) can be written

$$
\begin{aligned}
\sigma_{n}(\mathrm{P}, \mathrm{T})=\left(\begin{array}{c}
A_{\mathrm{P}} \\
n
\end{array}\right) \int_{-\xi_{\mathrm{T}}}^{\infty} d x\left(x+\xi_{\mathrm{T}}\right) \\
\quad \times\left[1-f_{\mathrm{P}}(x)\right]^{n} f_{\mathrm{P}}(x)^{A} \mathrm{P}^{-n},
\end{aligned}
$$

where the lower limit of integration can be put equal to zero since this introduces at most a one percent error even in the less favorable cases. We write

$$
\sigma_{n}(\mathrm{P}, \mathrm{T})=\left(\begin{array}{c}
A_{\mathrm{P}} \\
n
\end{array}\right)\left(\left\langle x_{n \mathrm{P}}\right\rangle+\xi_{\mathrm{T}}\right) N_{n \mathrm{P}},
$$

where $N_{n \mathrm{P}}$ is the normalization constant relative to the distribution $\left[1-f_{\mathrm{P}}(x)\right]^{n}\left[f_{\mathrm{P}}(x)\right]^{A} \mathrm{P}^{-n}$ and where $\left\langle x_{n \mathrm{P}}\right\rangle$ is the corresponding $x$ average.

The ratio

$$
\frac{\sigma_{n}(\mathrm{P}, \mathrm{T})}{\sigma_{n^{\prime}}(\mathrm{P}, \mathrm{T})}=\frac{\left(\begin{array}{c}
A_{\mathrm{P}} \\
n
\end{array}\right)}{\left(\begin{array}{c}
A_{\mathrm{P}} \\
n^{\prime}
\end{array}\right)} \frac{N_{n \mathrm{P}}}{N_{n^{\prime} \mathrm{P}}} \frac{\left\langle x_{n \mathrm{P}}\right\rangle+\xi_{\mathrm{T}}}{\left\langle x_{n^{\prime} \mathrm{P}}\right\rangle+\xi_{\mathrm{T}}}
$$

should be independent of $\mathrm{T}$ if weak factorization is valid. This is indeed the case for either $\left\langle x_{n p}\right\rangle \approx$ $\left\langle x_{n^{\prime} \mathrm{P}}\right\rangle$ or $\left\langle x_{n \mathrm{P}}\right\rangle$ and $\left\langle x_{n \mathrm{P}}^{\prime}\right\rangle \gg \xi_{\mathrm{T}}$ as can be seen from
TABLE II. The average $\left\langle x_{n \mathrm{P}}\right\rangle$ (see text) for the ${ }^{27} \mathrm{Al}$ and the ${ }^{107} \mathrm{Ag}$ projectiles for different values of the number $n$ of abraded nucleons.

\begin{tabular}{cccc}
\hline \hline & & \multicolumn{2}{c}{$\mathrm{P}={ }^{107} \mathrm{Ag}$} \\
$n$ & $\left\langle x_{n \mathrm{P}}\right\rangle$ & $n$ & $\left\langle x_{n \mathrm{P}}\right\rangle$ \\
\hline 1 & 8.4 & 1 & 11 \\
2 & 7.8 & 2 & 10.7 \\
3 & 7.4 & 3 & 10.3 \\
4 & 7.0 & 4 & 10 \\
5 & 6.7 & 5 & 9.6 \\
6 & 6.4 & 6 & 9.3 \\
\hline \hline
\end{tabular}

Tables I and II. It appears that weak factorization holds within $10-15 \%$ on an average. In the least favorable case ( $n$ very different from $n^{\prime}$ and $T={ }^{16} \mathrm{O}$ ) the weak factorization is broken by some $20 \%$.

We also looked at the ratio

$$
\frac{\sigma_{n}(\mathrm{P}, \mathrm{T})}{\sigma_{n}\left(\mathrm{P}, \mathrm{T}^{\prime}\right)}=\frac{\left\langle x_{n \mathrm{P}}\right\rangle+\xi_{\mathrm{T}}}{\left\langle x_{n \mathrm{P}}\right\rangle+\xi_{\mathrm{T}^{\prime}}}
$$

which should not depend upon $n$ if weak factorization is valid. This is realized if $\left\langle x_{n}\right\rangle$ is much larger than $\xi_{\mathrm{T}}$ whatever $n$ and $\mathrm{T}$ are. Tables $\mathrm{I}$ and II and Fig. 1 indicate that, once again, Eq. (10) is satisfied by $10 \%$ on an average, but this percentage becomes larger for the ${ }^{16} \mathrm{O}$ projectile when $\mathrm{T}$ is very different from $\mathrm{T}^{\prime}$.

Equation (10) and our tables tell that strong factorization is not so well satisfied as the weak one. Indeed the range of variation of $\left\langle x_{n p}\right\rangle$ becomes rather large when both $n$ and $\mathrm{P}$ are allowed to vary while it is much smaller when $n$ is varied with $P$ kept fixed [notice that relation (9) does not tell anything about the strong factorization property].

The weak and simple dependence of the fragmentation cross sections upon the details of the target can be traced back to the interplay between the exponential form of Eq. (4) and the relatively high value of $\sigma_{N N}$. Hence the projectile only "sees" the outer fringes of the target and one may consider that in the last resort the factorization arises from the relatively short mean free path of the high-energy nucleons in nuclear matter.

Helpful discussions with Professor C. Mahaux and Mr. G. Richelle are gratefully acknowledged. One of us (R.S.) would like to thank the Institut Interuniversitaire des Sciences Nucléaires for financial support. 
*Present address: Kellogg Radiation Laboratory, Cali-fornia Institute of Technology, Pasadena, California 91125.

${ }^{1}$ H. H. Heckman, D. E. Greiner, P. J. Lindstrom, and F. S. Bieser, Phys. Rev. Lett. 28, 926 (1972).

${ }^{2}$ P. J. Lindstrom, D. E. Greiner, H. H. Heckman, B. Cork, and F. S. Bieser, Lawrence Berkeley Laboratory Report No. LBL-3650 (unpublished).

${ }^{3}$ M. Bleszynski and C. Sander, Nucl. Phys. A326, 525 (1979).

${ }^{4}$ J. D. Bowman, W. J. Swiatecki, and C. F. Tsang, Lawrence Berkeley Laboratory Report No. LBL-2908 (unpublished).

${ }^{5} \mathrm{~J}$. Hüfner, K. Shäfer, and B. Shürmann, Phys. Rev. C 12,1888 (1975).

${ }^{6} \mathrm{~A}$ Abul-Magd, J. Hüfner, and B. Shürmann, Phys. Lett. 60B, 327 (1976).
${ }^{7}$ A. Abul-Magd and J. Hüfner, Z. Phys. A227, 379 (1976).

${ }^{8} \mathrm{~J}$. Hüfner, C. Sander, and G. Wolschin, Phys. Lett. 73B, 289 (1978).

${ }^{9} \mathrm{R}$. J. Glauber, in Lectures in Theoretical Physics, edited by W. E. Brittin and L. G. Dunham (Interscience, New York, 1959), Vol. 1.

${ }^{10}$ W. Czyz and L. C. Maximon, Ann. Phys. (N.Y.) 52, 59 (1969).

${ }^{11}$ O. Kofoed-Hansen, Nuovo Cimento A60, 621 (1969).

${ }^{12}$ J. Formánek, Nucl. Phys. B12, 441 (1969).

${ }^{13}$ G. Fäldt, Phys. Rev. D 2, 846 (1970).

${ }^{14}$ A. Tékou, Nucl. Phys. B46, 141, 152 (1972).

${ }^{15}$ G. Fäldt, H. Pilkuhn, and H. G. Schlaile, Ann. Phys. (N.Y.) 82,326 (1974).

${ }^{16}$ P. M. Fishbane and J. S. Trefil, Phys. Rev. D 10, 3005, 3228 (1974); Phys. Rev. Lett. 32, 396 (1974).

${ }^{17}$ V. Franco, Phys. Rev. Lett. 32, 911 (1974). 